\title{
Intermittent Program or Algorithm Execution
}

National Cancer Institute

\section{Source}

National Cancer Institute. Intermittent Program or Algorithm Execution. NCI Thesaurus.

Code C133603.

Problem associated with intermittent execution relating to program or algorithm. 